\title{
RESPONSE TO COMMENTS ON PAPER ON GRAIN BOUNDARY SCATTERING MODEL FOR METALS
}

\author{
F. WARKUSZ \\ Institute of Physics, Technical University of Wroctaw, ul. Wybrzeże Wyspiańskiego 27, 50-370 Wroctaw, \\ Poland.
}

(Received June 2, 1980; in revised form December 18, 1980; in final form February 11, 1981)

\begin{abstract}
The electrical conductivity of a polycrystalline metal film has been studied for a model in which the background scattering and grain boundary scattering are independent. The external surface electron scattering has been analyzed by assuming it to be independent of background scattering and thus the external surface scattering can be conveniently described with the Cottey method.
\end{abstract}

\section{INTRODUCTION}

An attempt has been recently made ${ }^{1}$ to give a new model of electric conduction in thick metal films. Some points from this model are discussed by Tellier and Tosser. ${ }^{2}$ The proposed model in paper 1 discussing scattering on grain boundary is similar to the Mayadas-Shatzkes model (Section 2). In Section 3 we shall prove, that to describe the external surface scattering in thin films we can use the Cottey method.

\section{GRAIN BOUNDARY ELECTRON SCATTERING}

In this Section we shall prove, that our model ${ }^{1}$ of electron scattering on grain boundaries is similar to the Mayadas-Shatzkes model ${ }^{3,4}$ and we shall also demonstrate, that the electron transmission coefficient $r$ has the value: $0 \leqslant r \leqslant 1$, similar to the electron reflection coefficient $R$ at a grain boundary, which has also the value: $0 \leqslant R \leqslant 1$.

The conductivity for metal films ${ }^{4}$ in the presence of both grain-boundary and background scattering is found from

$$
\sigma_{\mathrm{g}}=\frac{e^{2}}{4 \pi^{3}} \int \frac{\tau^{*} v^{2}}{\left|\nabla_{k} \varepsilon\right|} \mathrm{d} S_{F}
$$

where

$$
\begin{aligned}
& \tau^{*}=\frac{\tau}{1+\frac{\alpha}{q}} ; \quad \frac{1}{\tau^{*}}=\frac{1}{\tau}+\frac{1}{\tau} \alpha \frac{k_{F}}{\left|k_{x}\right|} \\
& \alpha=\frac{m s^{2} 2 \tau}{\hbar^{3} \mathrm{~d} k_{F}}=\frac{\lambda}{D} \frac{R}{1-R}
\end{aligned}
$$

$\lambda$ is the background mean free path, $D$ is the average grain diameter, and $R$ is the reflection coefficient

$$
\begin{aligned}
k_{X} & =k_{F}|\cos \gamma|=k_{F}|\cos \Phi \| \sin \theta|, \\
q & =\cos \gamma=\cos \Phi \sin \theta
\end{aligned}
$$

where $k_{F}-$ is the magnitude of the Fermi wavevector.

$$
\begin{aligned}
& \left|\nabla_{k} \varepsilon\right|^{-1}=\left(\frac{m}{2 \hbar^{2}}\right)^{1 / 2} \varepsilon^{-1 / 2} \\
& \mathrm{~d} S_{F}=\frac{2 m}{h^{2}} \varepsilon_{F} \sin \theta \mathrm{d} \theta \mathrm{d} \Phi
\end{aligned}
$$

Combining Eqs. (6), (5), (4), (3), (2), and Eq. (1), we obtain

$$
\sigma_{g}=\frac{2 e^{2} m^{2} v_{F}^{3} \tau}{h^{3}} \int_{0}^{2 \pi} \mathrm{d} \Phi \int_{0}^{\pi} \mathrm{d} \theta \frac{(\cos \Phi \sin \theta)^{2} \sin \theta}{1+\frac{\alpha}{|\cos \Phi \| \sin \theta|}}
$$

Assuming that $\sigma_{0}$ is the conductivity in the absence of grain boundaries (bulk conductivity), we obtain

$$
\begin{gathered}
\sigma_{0}=\frac{8 \pi e^{2} m^{2} \tau v_{F}^{3}}{3 h^{3}} \\
\frac{\sigma_{g}}{\sigma_{0}}=\frac{3}{4 \pi} \int_{0}^{2 \pi} \mathrm{d} \Phi \int_{0}^{\pi} \mathrm{d} \theta \frac{(\cos \Phi \sin \theta)^{2} \sin \theta}{1+\frac{\alpha}{|\cos \Phi \| \sin \theta|}} \equiv G(\alpha)
\end{gathered}
$$

or

$$
\frac{\sigma_{g}}{\sigma_{0}}=\frac{3}{4 \pi} \int_{0}^{2 \pi} \mathrm{d} \Phi \int_{0}^{\pi} \mathrm{d} \theta \frac{(\cos \Phi \sin \theta)^{2} \sin \theta}{H(\Phi, \theta, \alpha)}
$$


where

$$
H(\Phi, \theta, \alpha)=1+\frac{\alpha}{|\cos \Phi \| \sin \theta|}
$$

Equation (9) has been obtained by Mayadas and Shatzkes ${ }^{4}$ by considering the total resistivity of a thin metal film in which three types of electron scattering mechanisms are simultaneously present: an isotropic background scattering, scattering due to a distribution of planar potentiais grain boundaries, and scattering due to external surfaces. The intrinsic or bulk resistivity is obtained by solving the Boltzmann equation in which both grain-boundary and background scatterings are accounted for. The total resistivity is obtained by imposing boundary conditions due to the external surfaces as in the Fuchs ${ }^{5,6}$ theory using the Boltzmann equation.

Assuming that

$$
\sigma_{f}=\frac{1}{E a} \int_{0}^{a} \mathrm{~d} z\left\{\frac{-2 e m^{3}}{h^{3}} \int v_{x} f(\mathbf{v}) \mathrm{d} v_{x} \mathrm{~d} v_{y} \mathrm{~d} v_{z}\right\}
$$

and imposing boundary conditions, due to the external surface as in the Fuchs-Sondheimer theory ${ }^{5,6}$, we obtain:

$$
\begin{gathered}
\sigma_{f}=\frac{1}{E a} \int_{0}^{a} \mathrm{~d} z\left\{-\frac{2 e^{2} m^{2} E}{h^{3}} \int_{0}^{\infty} \mathrm{d} v \int_{0}^{2 \pi} \mathrm{d} \Phi \int_{0}^{\pi / 2} \mathrm{~d} \theta v^{3} \cos ^{2} \Phi\right. \\
\sin ^{3} \theta \tau^{*} \frac{\partial f_{0}}{\partial v}\left[1-\frac{1-p}{1-p \exp \left(-a / \tau^{*} v \cos \theta\right)}\right] \\
\quad \times \exp \left(-\frac{z}{\tau^{*} v \cos \theta}\right)-\frac{2 e^{2} m^{2} E}{h^{3}} \int_{0}^{\infty} \mathrm{d} v \int_{0}^{2 \pi} \mathrm{d} \Phi \int_{\pi / 2}^{\pi} \\
\mathrm{d} \theta v^{3} \cos ^{2} \Phi \sin ^{3} \theta \tau^{*} \frac{\partial f_{0}}{\partial v}\left[1-\frac{1-p}{1-p \exp \left(a / \tau^{*} v \cos \theta\right)}\right] \\
\left.\exp \left(\frac{a-z}{\tau^{*} v \cos \theta}\right)\right\}
\end{gathered}
$$

Assuming that

$\int_{0}^{\infty} v^{3} \frac{\partial f_{0}}{\partial v} \mathrm{~d} v=-v_{F}^{3} ; k_{F}=\frac{k_{x}}{|\cos \Phi \| \sin \theta|} ; \tau v=\lambda$

$\frac{1}{\tau^{*}}=\frac{1}{\tau}+\frac{1}{\tau} \frac{\alpha}{|\cos \Phi \| \sin \theta|} ; \quad \tau^{*}=\frac{\tau}{H(\Phi, \theta, \alpha)}$

where

$$
H(\Phi, \theta, \alpha)=1+\frac{\alpha}{|\cos \Phi \| \sin \theta|}
$$

the Eq. (11) becomes

$$
\begin{gathered}
\sigma_{f}=\frac{4 e^{2} m^{2} v_{F}^{3} \tau}{h^{3}} \int_{0}^{2 \pi} \mathrm{d} \Phi \int_{0}^{\pi / 2} \mathrm{~d} \theta \frac{\cos ^{2} \Phi \sin ^{3} \theta}{H(\Phi, \theta, \alpha)} \\
+-\frac{4 e^{2} m^{2} v_{F}^{3} \tau}{h^{3}} \frac{\lambda}{a}(1-p) \int_{0}^{2 \pi} \mathrm{d} \Phi \int_{0}^{\pi / 2} \mathrm{~d} \theta \\
\frac{\cos ^{2} \Phi \sin ^{3} \theta \cos \theta}{H^{2}(\Phi, \theta, \alpha)} \frac{1-\exp \left(-\frac{a H(\Phi, \theta, \alpha)}{\lambda \cos \theta}\right)}{1-p \exp \left(-\frac{a H(\Phi, \theta, \alpha)}{\lambda \cos \theta}\right)}
\end{gathered}
$$

Considering Eq. (8)

$$
\begin{array}{r}
\frac{\sigma_{f}}{\sigma_{0}}=\frac{3}{2 \pi} \int_{0}^{2 \pi} \mathrm{d} \Phi \int_{0}^{\pi / 2} \mathrm{~d} \theta \frac{\cos ^{2} \Phi \sin ^{3} \theta}{H(\Phi, \theta, \alpha)}-\frac{3}{2 \pi} \frac{\lambda}{a} \\
\times(1-p) \int_{0}^{2 \pi} \mathrm{d} \Phi \int_{0}^{\pi / 2} \mathrm{~d} \theta \frac{\cos ^{2} \Phi \sin ^{3} \theta \cos \theta}{H^{2}(\Phi, \theta, \alpha)} \\
\times \frac{1-\exp \left(-\frac{a H(\Phi, \theta, \alpha)}{\lambda \cos \theta}\right)}{1-p \exp \left(-\frac{a H(\Phi, \theta, \alpha)}{\lambda \cos \theta}\right)}
\end{array}
$$

The first term on the right hand side of Eq. (15) is the grain boundary scattering function (Eq. (9)). The equation (15) is often described as

$$
\frac{\sigma_{f}}{\sigma_{0}}=G(\alpha)-A(k, p, \alpha)
$$

$G(\alpha)$ is a grain-boundary function and can be expressed by Eq. (9) or by Eq. (1). Considering that

$$
\cos \Phi \sin \theta=\cos \gamma=q,
$$

(see Figure 1) and Eq. (2), we have that Eq. (9) can be transformed to

$$
\frac{\sigma_{g}}{\sigma_{0}}=\frac{3}{2} \frac{1}{\tau} \int_{-1}^{1} \tau^{*}(q) q^{2} \mathrm{~d} q=3 \int_{0}^{1} \frac{q^{3}}{\alpha+q} \mathrm{~d} q
$$

Eq. (17) was also obtained by Mayadas and Shatzkes., The solution of the integral in Eq. (17) includes the following expression

$$
\frac{\sigma_{g}}{\sigma_{0}}=1-\frac{3}{2} \alpha+3 \alpha^{2}-3 \alpha^{3} \ln \left(1+\frac{1}{\alpha}\right) \equiv G(\alpha)
$$

Let us examine the equations (9) and (17), because these equations refer to electrical conductivity for the film, for which we consider the background scattering and the grain boundary scattering. The model given in paper ${ }^{1}$ considers similar problems and as it will be further shown, the effects of electron scattering can also be calculated by means of Matthiessen's rule starting 


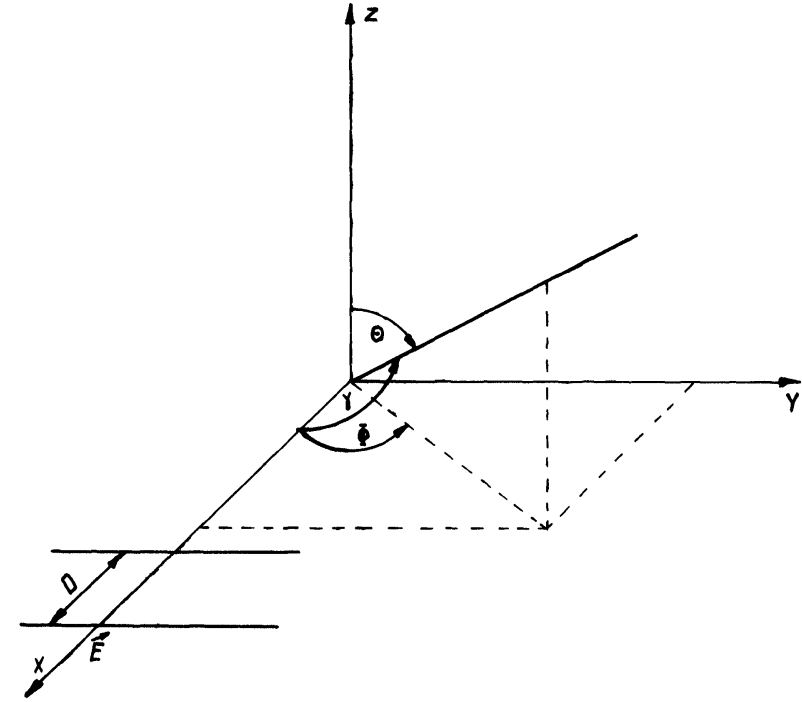

FIGURE 1 Geometry of the scattering planes model

from the electron mean free path related to a particular type of scattering (bulk and grain boundaries).

Similarly, considering that ${ }^{1}$

$$
\frac{1}{\tau^{*}(D, r)}=\frac{1}{\tau}+\frac{1}{\tau_{D}}
$$

where

$$
\tau_{D}=\frac{D}{v_{F}|\cos \Phi \| \sin \theta| \ln 1 / r}
$$

$D$ is the average grain diameter (i.e. $D=\sum_{i} \mathrm{~d}_{i} / n$ )

$$
V_{X}=V_{F} \cos \Phi \sin \theta=V_{F} \cos \gamma \quad \text { (see Fig. 1) }
$$

we obtain

$$
\tau^{*}(D, r)=\frac{\tau}{1+\frac{|\cos \Phi \| \sin \theta|}{v}}
$$

where

$$
v=\frac{D}{\lambda \ln 1 / r} ; \quad \lambda=\tau v_{F}
$$

Considering the relation

$$
\sigma_{g}=-\frac{2 e m^{3}}{E h^{3}} \int v_{x} f_{1} \mathrm{~d} v_{x} \mathrm{~d} v_{y} \mathrm{~d} v_{z}
$$

and the Boltzmann equation

$$
\frac{f_{1}}{\tau^{*}(D, r)}=\frac{e E}{m} \frac{\partial f_{0}}{\partial v_{x}}
$$

we obtain

$$
\frac{\sigma_{g}}{\sigma_{0}}=\frac{3}{4 \pi} \int_{0}^{2 \pi} \mathrm{d} \Phi \int_{0}^{\pi} \mathrm{d} \theta \frac{(\cos \Phi \sin \theta)^{2} \sin \theta}{1+\frac{|\cos \Phi \| \sin \theta|}{v}} \equiv F(v)
$$

For the cylindrical grain boundaries, Eq. (26) assumes a simple form and was derived previously. ${ }^{1,7}$

Comparing Eqs. (26) and (9), we obtain

$$
\frac{\cos \Phi \sin \theta}{v} \sim \frac{\alpha}{\cos \Phi \sin \theta}
$$

Considering that ${ }^{3,4}$

$$
\alpha=\frac{\lambda}{D} \frac{R}{1-R}
$$

and $^{1,7}$

$$
v=\frac{D}{\lambda \ln 1 / r}
$$

we obtain

$$
\frac{R}{1-R} \sim \cos ^{2} \Phi \sin ^{2} \theta \ln 1 / r
$$

As $0 \leqslant R \leqslant 1$ thus also $0 \leqslant r \leqslant 1$. Similarly we have $\mathrm{e}^{3,4}$

$$
\alpha=\frac{m}{\hbar^{3} d} \frac{s^{2} 2 \tau}{k_{F}}=\frac{m}{\hbar^{3} d} \frac{s^{2} 2 \tau}{k_{x}} \cos \Phi \sin \theta
$$

and

$$
v \sim \frac{\cos ^{2} \Phi \sin ^{2} \theta}{\alpha} \sim\left(\frac{m}{\hbar^{3} d} \frac{s^{2} 2 \tau}{k_{x}}\right)^{-1} \cos \Phi \sin \theta
$$

If no electrons are travelling through the grain boundary then $r=0(R=1)$. If all electrons pass the grain boundary, then $r=1(R=0)$. If we introduce Eq. (32) into Eq. (26), then we obtain Eq. (9) or Eq. (17). This is the Mayadas-Shatzkes expression. Tellier and Tosser ${ }^{2}$ assume that the proposed model is true only for $r \approx 1$. As it been shown above, Eq. (26) is valid if $r$ takes any value in the range $0 \leqslant r \leqslant 1$. Considering Eqs. (1) and (22) and $q=\cos \Phi \sin \theta$, we obtain

$$
\sigma_{g}=\frac{e^{2}}{4 \pi^{3}} \int \frac{\tau^{*}(D, r) v_{x}^{2}}{\mid \nabla_{k} \varepsilon \|} \mathrm{d} S_{F}=3 \sigma_{0} v \int_{0}^{1} \frac{q^{2}}{v+q} \mathrm{~d} q
$$

Integration of Eq. (33) gives

$$
\frac{\sigma_{g}}{\sigma_{0}}=\frac{3}{2} v-3 v^{2}+3 v^{3} \ln \left(1+\frac{1}{v}\right) \equiv F(v)
$$

Eq. (34) reduces to the approximate expressions for the large grain and for the very thin grain respectively. 


$$
\begin{array}{ll}
\frac{\sigma_{g}}{\sigma_{0}}=1-\frac{3}{4 v}+\frac{1}{5 v^{2}}-\cdots & \text { for } v>1 \\
\frac{\sigma_{g}}{\sigma_{0}}=\frac{3}{2} v-3 v^{2}+\cdots & \text { for } v<1
\end{array}
$$

and

$$
\frac{\sigma_{g}}{\sigma_{0}}=0.5793 \quad \text { for } v=1
$$

Thus it would seem that the new model proposed recently ${ }^{1}$ is valid and the expressions for the Mayadas-Shatzkes equation (Eq. (9) and Eq. (17)), together with the alternative expressions (Eq. (26) and Eq. (33)), respectively are relatively simple. ${ }^{2}$

Recently, Tellier and Tosser ${ }^{8}$ have reported a statistical model for thin metallic films in which the grain boundaries are perpendicular to the $\mathrm{x}$-direction and y-direction, assuming that the transmission coefficent (denoted by $t$ in their papers) can assume values less than unity. Such a statistical model is a special case of the present model (Eq.(26)).

\section{EXTERNAL SURFACE ELECTRON SCATTERING}

Let us consider both the Fuchs method ${ }^{5,6}$ and the Cottey method ${ }^{9}$ used to describe electrical conductivity for metallic films in which the background electron scattering and the external surface scattering are examined. The surface reflection parameter $p$ will be discussed in detail. We consider some of the assumptions made by Fuchs ${ }^{5,6}$ and the present author, to describe the external size-effect. The Fuchs theory assumes that the relaxation time of bulk material is independent of the film thickness and the $p_{\text {Fuchs }}$ parameter.

$$
f_{1}=f_{1}(t=0) \exp \left(-\frac{t}{\tau}\right) ; \quad \tau=\text { const }
$$

where the function $f_{1}$ depends on the space variables only through $\mathrm{z}$.

$$
\frac{\partial f_{1}}{\partial z} \neq 0
$$

The boundary conditions at the surfaces of the film are described in general, as follows

$$
f_{1}\left(v_{z}, z\right)=p_{F} f_{1}\left(-v_{z}, z\right)
$$

The electric field $E$ is supposed to be in the x-direction and the Boltzmann equation reduces to

$$
\frac{\partial f_{1}}{\partial z}+\frac{f_{1}}{\tau v_{z}}=\frac{e E}{m v_{z}} \frac{\partial f_{0}}{\partial v_{x}}
$$

Carrying out an integration over $z$, we obtain for the effective conductivity of the film

$$
\sigma_{f}=\frac{1}{E a} \int_{0}^{a} \mathrm{~d} z\left\{-\frac{2 e m^{3}}{h^{3}} \int v_{x} f_{1} \mathrm{~d} v_{x} \mathrm{~d} v_{y} \mathrm{~d} v_{z}\right\}
$$

In our model ${ }^{8,9}$ of the electron surface scattering we consider that

$f_{1}=f_{1}(t=0) \exp \left(-\frac{t}{\tau(a, p)}\right) ; \quad \tau(a, p) \neq$ const

and

$$
\frac{\partial f_{1}}{\partial z}=0
$$

This term is neglected in the Boltzmann equation. We assume, that the external surface scattering and background scattering are independent and the relaxation time $\tau(a, p)$ for both scatterings equals:

$$
\frac{1}{\tau(a, p)}=\frac{1}{\tau}+\frac{1}{\tau_{a}}
$$

Hence the theoretical expression for $\tau(a, p)$ is

$$
\tau(a, p)=\frac{\tau a}{a+\tau \ln \left(\frac{1}{p}\right) v_{F} \cos \theta}
$$

The $p$-parameter can also be conveniently described by the Cottey model, $p_{\text {Cottey }}$.

If there is an electric field, $E$, in the $x$-direction, the linearized Boltzmann equation is

$$
\frac{f_{1}}{\tau(a, p)}=\frac{e E}{m} \frac{\partial f_{0}}{\partial v_{x}}
$$

and the conductivity of the film is found to be

$$
\sigma_{f}=-\frac{2 e m^{3}}{E h^{3}} \int v_{x} f_{1} \mathrm{~d} v_{x} \mathrm{~d} v_{y} \mathrm{~d} v_{z}
$$

In general, the parameter $p_{\text {Fuchs }}=p_{\text {Cottey }}$ if $p_{\text {Fuchs }}$ is near 1 and $p_{\text {Cottey }}$ is near 1 . We, however, are concerned with $0 \leqslant p_{\text {Fuchs }} \leqslant 1$ and $0 \leqslant p_{\text {Cottey }} \leqslant 1$. The Fuchs parameter is the probability that an electron will be specularly reflected upon scattering from a film surface. Our parameter $p$ is the probability that an electron will be reflected upon scattering from a film surface. If $p_{\text {Fuchs }}=0$, then all the models agree for diffuse scattering ${ }^{6}$ - for example:

$$
\begin{array}{r}
\sigma_{f}=\sigma_{0}\left\{1-\frac{3}{8} \frac{\lambda}{a}+\frac{3}{2} \frac{\lambda}{a} \int_{0}^{\pi / 2} \sin \theta\left(\cos ^{3} \theta-\cos ^{5} \theta\right)\right. \\
\left.\exp \left(-\frac{a}{\lambda \cos \theta}\right) \mathrm{d} \theta\right\}
\end{array}
$$


If $p=0$, in our model no conduction electrons are reflected from an external film surface and the film conductivity $\sigma_{f}$ equals zero.

Considering the background, grain-boundary and external-surface scatterings in our model, we obtain

$$
\frac{\sigma_{f}}{\sigma_{0}}=\frac{3}{4 \pi} \int_{0}^{2 \pi} \mathrm{d} \Phi \int_{0}^{\pi} \mathrm{d} \theta \frac{\cos ^{2} \Phi \sin ^{3} \theta}{1+\frac{|\cos \theta|}{\mu}+\frac{|\cos \Phi||\sin \theta|}{v}}
$$

where

$$
\mu=\frac{a}{\lambda \ln 1 / p} ; \quad v=\frac{D}{\lambda \ln 1 / r}
$$

This integral has been calculated explicitly for cylindrical grain boundaries. ${ }^{10}$

Recently a three-dimensional model of grain boundaries has been proposed by Pichard, Tellier and Tosser. ${ }^{11}$ This is an extension of the present grain scattering model since it is possible that the reflection parameter $p$, and the transmission coefficient $t$ can assume values less than unity. This contrasts with previous comments ${ }^{2}$ (see also Refs. 11 and 12).

\section{REFERENCES}

1. F. Warkusz, Electrocomp. Sc. Technol., 5, 197 (1978).

2. C. R. Tellier and A. J. Tosser, Electrocomp. Sc. Technol., 6, 37 (1979).

3. A. F. Mayadas, M. Shatzkes and J. F. Janak, Appl. Phys. Let., 14, 345 (1969).

4. A. D. Mayadas and Shatzkes, Phys. Rev. B, 1,1382 (1970).

5. K. Fuchs, Pro. Camb. Philos. Soc., 34, 100 (1938)

6. E. H. Sondheimer, Adv. Phys., 1, 1 (1952).

7. F. Warkusz, Thin Solid Films, 62, 247 (1979).

8. C. R. Pichard, A. J. Tosser and C. R. Tellier, Electrocomp. Sc. Technol., 6, 87 (1980).

9. A. A. Cottey, Thin Solid Films, 1, 297 (1968).

10. F. Warkusz, Acta. Phys., Pol. A, 54, 31 (1978).

11. C. R. Pichard, C. R. Tellier and A. J. Tosser, Thin Solid Films, 62, 189 (1979).

12. C. R. Tellier and A. J. Tosser, Thin Solid Films, 70, 225 (1980). 

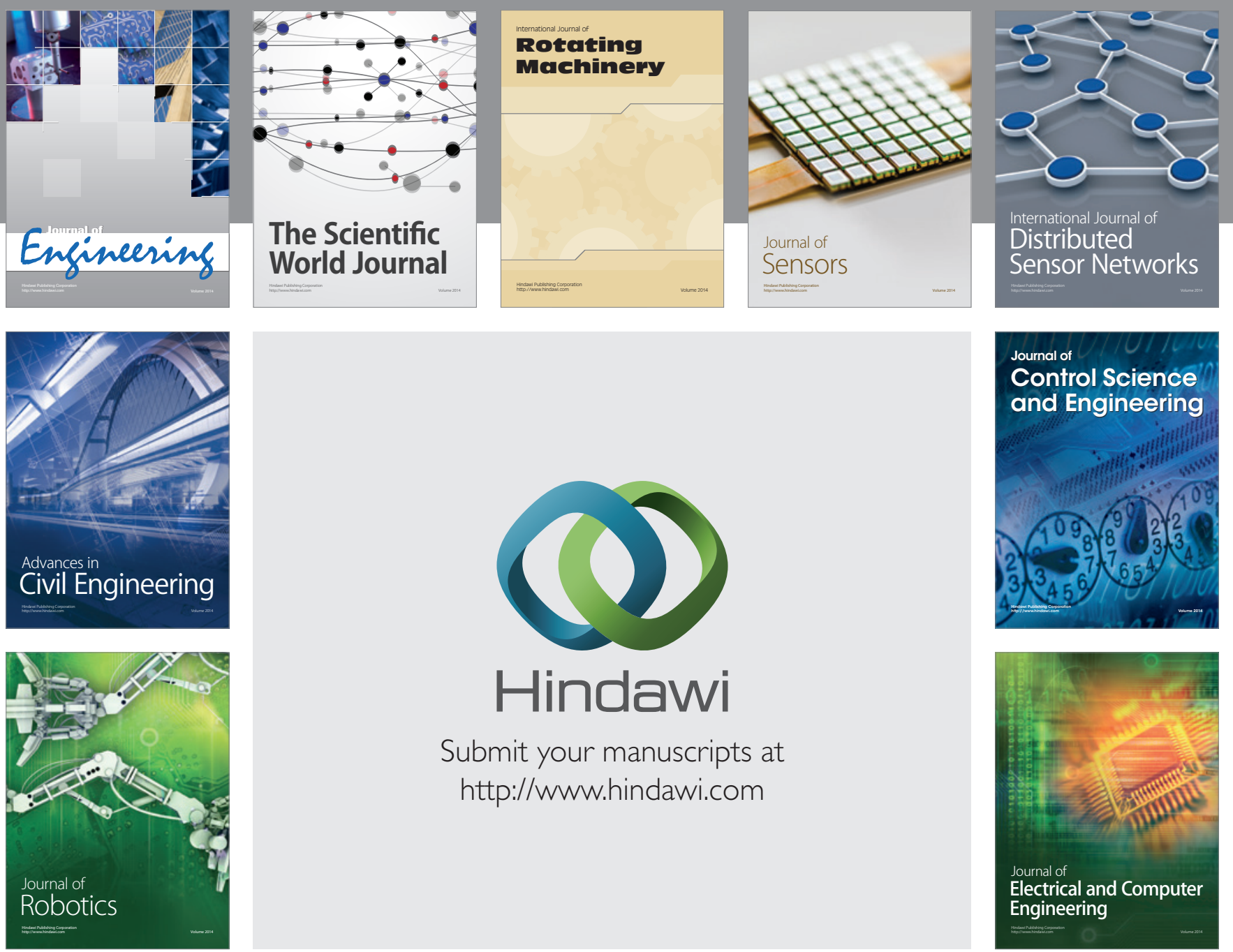

Submit your manuscripts at

http://www.hindawi.com
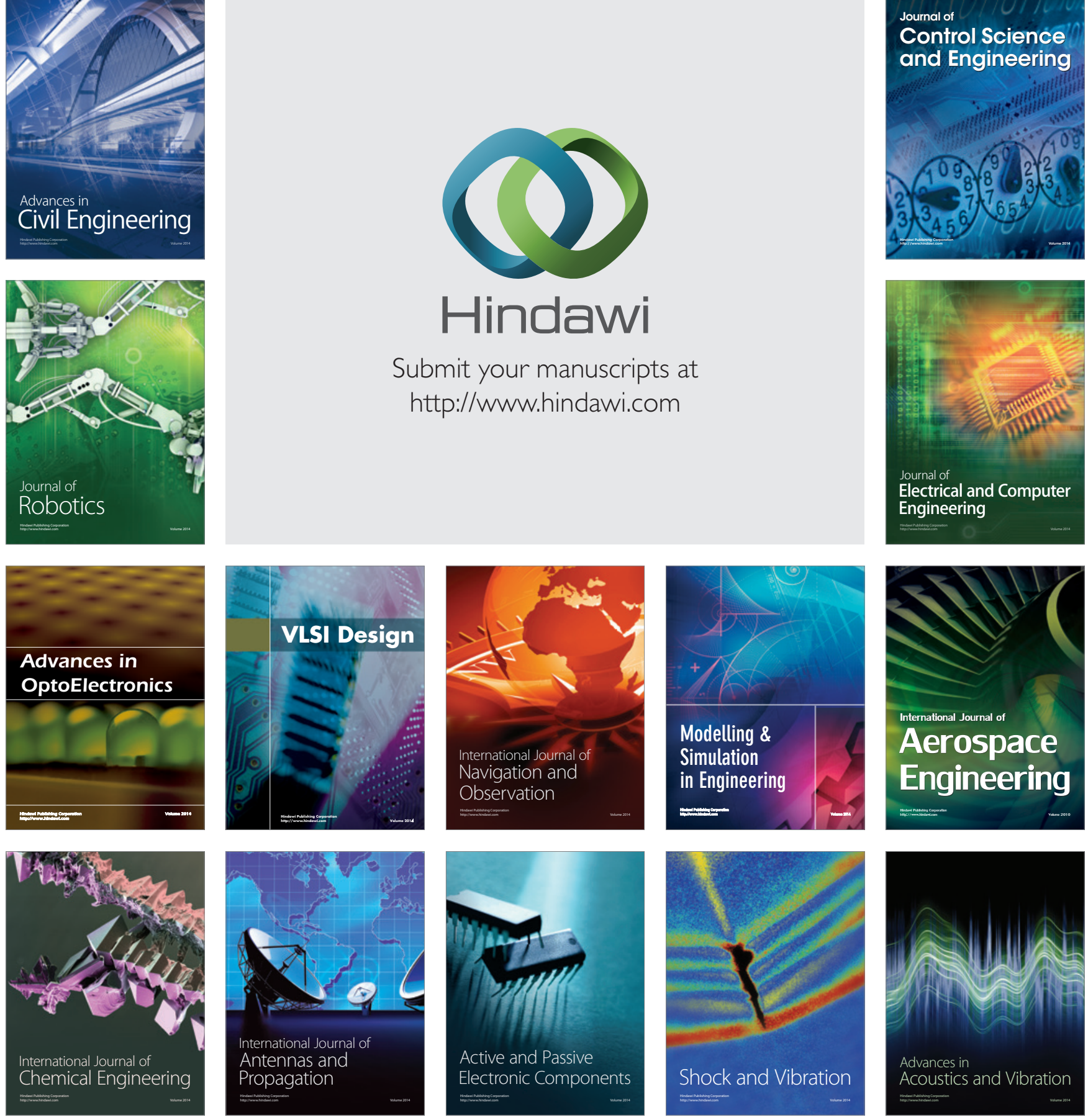